\title{
Understanding patient engagement in health system decision-making: a co-designed scoping review
}

Tamara L. McCarron ${ }^{1,2^{*}}$ D, Karen Moffat ${ }^{3}$, Gloria Wilkinson ${ }^{3}$, Sandra Zelinsky ${ }^{3}$, Jamie M. Boyd ${ }^{1,2}$, Deborah White ${ }^{4}$, Derek Hassay ${ }^{5}$, Diane L. Lorenzetti ${ }^{1,2,6}$, Nancy J. Marlett ${ }^{1,2}$ and Thomas Noseworthy ${ }^{1,2}$

\begin{abstract}
Background: With healthcare striving to shift to a more person-centered delivery model, patient and family involvement must have a bigger role in shaping this. While many initiatives involving patients and family members focus on self-care, a broader understanding of patient participation is necessary. Ensuring a viable and sustainable critical number of qualified patients and family members to support this shift will be of utmost importance. The purpose of this study was to understand how health systems are intentionally investing in the training and skill development of patients and family members.
\end{abstract}

Methods: Patient co-investigators and researchers conducted a scoping review of the existing literature on methods adopted by healthcare systems to build the skills and capacity of patients to participate in healthcare decision-making using a recognized methodological framework. Six electronic databases were searched to identify studies. Two independent reviewers screened titles and abstracts and full-text papers for inclusion. The research team independently extracted data. Any disagreements were resolved by achieving consensus through discussion. Quantitative and qualitative content synthesis, as well as a quality assessment, was conducted.

Results: After eliminating duplicates, the search resulted in 9428 abstracts. Four hundred fifty-eight articles were reviewed and 15 articles were included. Four themes emerged: forums (33\%), patient instructors (20\%), workshops (33\%), and co-design (13\%). Four of the identified studies measured the impact and overall effectiveness of the respective programs. Examples of how patient and family members were supported (invested in) included advocacy training to support future involvement in engagement activities, a training program to conduct patient-led research, involvement in an immersive experience-based co-design initiative, and involvement in training pharmacy students. Overall, these studies found positive outcomes when patients and family members were recipients of these opportunities.

Conclusions: The results of this scoping review demonstrate that an evidence base around programs to advance patient engagement is largely absent. An opportunity exists for further research to identify strategies and measures to support patient engagement in healthcare decision-making.

Keywords: Patients, Capacity building, Patient participation, Patient-centered, Decision-making

\footnotetext{
* Correspondence: tImccarr@ucalgary.ca

${ }^{1}$ The Department Community Health Sciences, Teaching Research and

Wellness Building, 3280 Hospital Drive NW, Calgary, Alberta T2N 4N1, Canada

${ }^{2} \mathrm{O}$ Brien Institute for Public Health, Teaching Research and Wellness Building,

3280 Hospital Drive NW, Calgary, Alberta T2N 4N1, Canada

Full list of author information is available at the end of the article
}

(c) The Author(s). 2019 Open Access This article is distributed under the terms of the Creative Commons Attribution 4.0 International License (http://creativecommons.org/licenses/by/4.0/), which permits unrestricted use, distribution, and

reproduction in any medium, provided you give appropriate credit to the original author(s) and the source, provide a link to the Creative Commons license, and indicate if changes were made. The Creative Commons Public Domain Dedication waiver (http://creativecommons.org/publicdomain/zero/1.0/) applies to the data made available in this article, unless otherwise stated. 


\section{Background}

The concept of including patients in health and health system decision-making has been around for over 50 years [1]. Patient involvement in various aspects of healthcare, from improving healthcare quality to promoting patient safety, has emerged as a critical priority, but understanding how best to engage patients is not well understood [2-10]. This knowledge gap results in frustrating barriers for decision-makers looking to draw transferable lessons to inform the design of patient engagement programs and processes [11-13]. Coupled with challenges to the sustainability of healthcare and the need for innovative solutions, patient engagement has become central to improving both quality and delivery of services [14]. Research has demonstrated that patients who take part in their healthcare decisions are likely to also have better health outcomes [15]. Following this line of logic, we can assume patients who actively engage in opportunities to improve healthcare decisionmaking may have gaps in the education and the training required to participate as an equal partner. While many initiatives focus on patient self-care, a broader approach to patient participation is necessary to support the effective restructuring of healthcare delivery. This requires a critical number of qualified patients and family members who not only want to engage, but who are also qualified and confident to work in partnership with healthcare professionals and other stakeholders. This involves harnessing the skills and further building the capabilities of patients to support their participation in healthcare decision-making across the entire health system $[14,16-18]$. This area of patient engagement is not well defined, and it is unclear what strategies are currently being implemented to promote the active engagement of patients in building their skills and capabilities. While there have been systematic reviews published that have explored patient engagement in research, these reviews have primarily focused on improving self-care [19] and improving shared decision-making [20]. Given this gap in understanding, we conducted a scoping review to systematically map out the research in this area. The objective of this study is to understand how health systems are intentionally investing in building the capacity and ability of patients to meaningfully participate in all aspects of healthcare decision-making. The following research question was formulated: How do health systems develop the ability and skills of patients and family members to engage in healthcare decision-making?

\section{Methods}

\section{Protocol and research question}

This scoping review protocol was developed using the methodological framework proposed by Arksey and O'Malley [21] and further enhanced by Levac et al. [22].
This review follows a six-stage methodological framework following these steps: (1) identify the research question, (2) identify relevant studies, (3) study selection, (4) charting the data, (5) collating, summarizing and reporting the results, and 6) stakeholder consultation $[21,22]$. Despite some contention within the academic community as to whether the quality assessment should be conducted or not, quality assessment of included studies was completed [23, 24]. We used a modified SPICE (setting, population/perspective, intervention, comparison and evaluation) methodology to develop our research question [25]. Our protocol was drafted a priori using the Preferred Reporting Items for Systematic Reviews and Meta-analysis Protocols (PRISMA-P). The final protocol was posted on the Open Science Framework (https://osf.io/2ta74/). This protocol was uploaded on 7 September 2018 but is not registered or otherwise published. This review was completed in accordance with the scoping review reporting guidelines (PRISMA-ScR) [26].

\section{Co-design and patient co-investigators}

This study utilized a co-design methodology, whereby members of the public, in this case, the patient and family community, were involved in the design of the research project from genesis to completion, including question development, data extraction, and interpretation [27, 28]. These patients were recruited based on their prior experiences participating in healthcare system decision-making and their ability to commit to each phase of this project. Three patient co-investigators and two researchers formed the project team. Co-investigators were given information 3 weeks prior to the meeting, highlighting the project goals and objectives and the high-level requirements needed to complete each step of the scoping review. At the initial meeting, the team discussed the research question and what was meant by the term "investment," and determined that it was important to consider not only the traditional understanding of investing, which is primarily financial in nature (i.e., payment or expenses), but also the act of devoting time, effort, or energy to an endeavor.

\section{Information sources and search strategy}

Search terms were debated with this enhanced understanding of investment in mind, and the project team created an exhaustive and wide-reaching list of search terms to adequately describe possible methods used by health systems to invest in patients. We designed the search strategy in collaboration with a librarian, to be broad and inclusive. The search strategy combined terms from three distinct themes: (1) investments (e.g., educating, learning, training), (2) influences (e.g., decision-making, self-efficacy, and innovation), and (3) areas of patient involvement (e.g., governance and co-design). The researcher 
and librarian systematically searched CINAHL, MEDLINE, EMBASE, Education Research Complete, Business Source Complete, and PsycINFO for studies published between January 1, 2000, and July 30, 2016. We limited the search strategy to studies written in English. The final search strategy for MEDLINE can be found in Additional file 1.

\section{Eligibility criteria}

Studies were included if they (1) had an adult patient/ consumer focus, (2) contained a description of an investment, (3) focused on programs/activities/events that were determined to have an impact on the participation of patients in healthcare, (4) showcased how patients/ consumers engaged with other patients or the health system, and (5) incorporated investments that enable patients/consumers to participate in various healthcare roles. Studies were excluded if they (1) focused on investments to improve self-care; (2) did not involve or engage patients; (3) focused on children, animals, or family members; (4) did not report outcomes; or (5) were opinion pieces or letters to the editor.

\section{Study identification}

All search results were merged into a reference management software program (EndNote X7).

The first 200 abstracts acted as a calibration process, enabling the project team to review and revise the inclusion criteria.

In order to best utilize the patient co-investigators, the first 1700 title and abstracts were reviewed (500 per patient co-investigator, duplicate review by the first author). The project team came together to review and discuss the results of the title/abstract review which provided further insights into the perspectives of the patient co-investigators and assisted the researcher and second reviewer with the approach taken to the remaining title and abstracts. Disagreements were resolved through consensus or by a third reviewer.

The first author provided the patient co-investigators with a training session on how to locate the articles flagged for review within the electronic databases. All articles were pulled for full-text review by the patient co-investigators.

The project team met again as a group to discuss and adjudicate the first 20 full-text articles selected for review. Any questions were answered, and the remainder of the steps was discussed. Selected articles were reviewed in duplicate by both patient investigators and the first author. As with the abstract review, any disagreements were resolved by a third reviewer.

\section{Quality assessment}

The Mixed Methods Appraisal Tool (MMAT) developed by McGill University was used to assess the quality of the identified studies [29]. This tool was chosen for its ease of use, and ability to assess a diversity of study designs [30]. The tool is comprised of two parts (an initial screening section followed by a series of four questions) to simultaneously appraise and describe the methodological quality of mixed, qualitative, and quantitative study designs [29]. In part 2 of the tool, retained studies that are qualitative or quantitative in design can result in scores ranging from $25 \%$ (when no criterion is met) to $100 \%$ (having met all 4 criteria). Mixed methods studies can result in scores ranging from 25\% (when no criterion is met) to $100 \%$ (having met three criteria). We assume a low-quality study as being one that only meets $25 \%$ (or 1 of 4 criteria), medium quality (meets 2 of 4 criteria), and high quality (meets 3 of 4 criteria). The quality of included studies was assessed in duplicate by two researchers. Studies were not excluded based on quality.

\section{Data extraction (charting)}

Frequencies and percentages were calculated to describe the data. A predefined data extraction (charting) sheet was developed by the study team. The research team independently extracted data and discussed the results and continuously updated the data-charting form in an iterative process. Any disagreements were resolved by achieving consensus through discussion.

We extracted data on article characteristics (e.g., country of origin, author, outcomes), participant characteristics (e.g., type of participant and number), investment characteristics (type of investment and description), and patient motivations (if included), see Additional file 2.

\section{Data synthesis}

The research team sorted each of the studies into "investment" themes by using a modified constant comparative method developed by Glaser [31]. This method required that the research team placed each study into an investment theme; comparing each new study to the previous to determine if there was a new theme. This process was repeated until all studies were placed into a unique theme.

\section{Results}

The search resulted in 12,170 articles (Fig. 1). Duplicates $(n=2732)$ were removed and 9438 articles underwent title and abstract review. Four hundred fifty-eight articles were selected for full-text review. Of these, 15 studies were included in this scoping review, see Fig. 1.

\section{Study characterization}

Of the 15 included studies, most $(n=8)$ were published after 2012 (Table 1). Studies were primarily published in the UK $(n=7)$ followed by the US $(n=3)$ with the fewest 


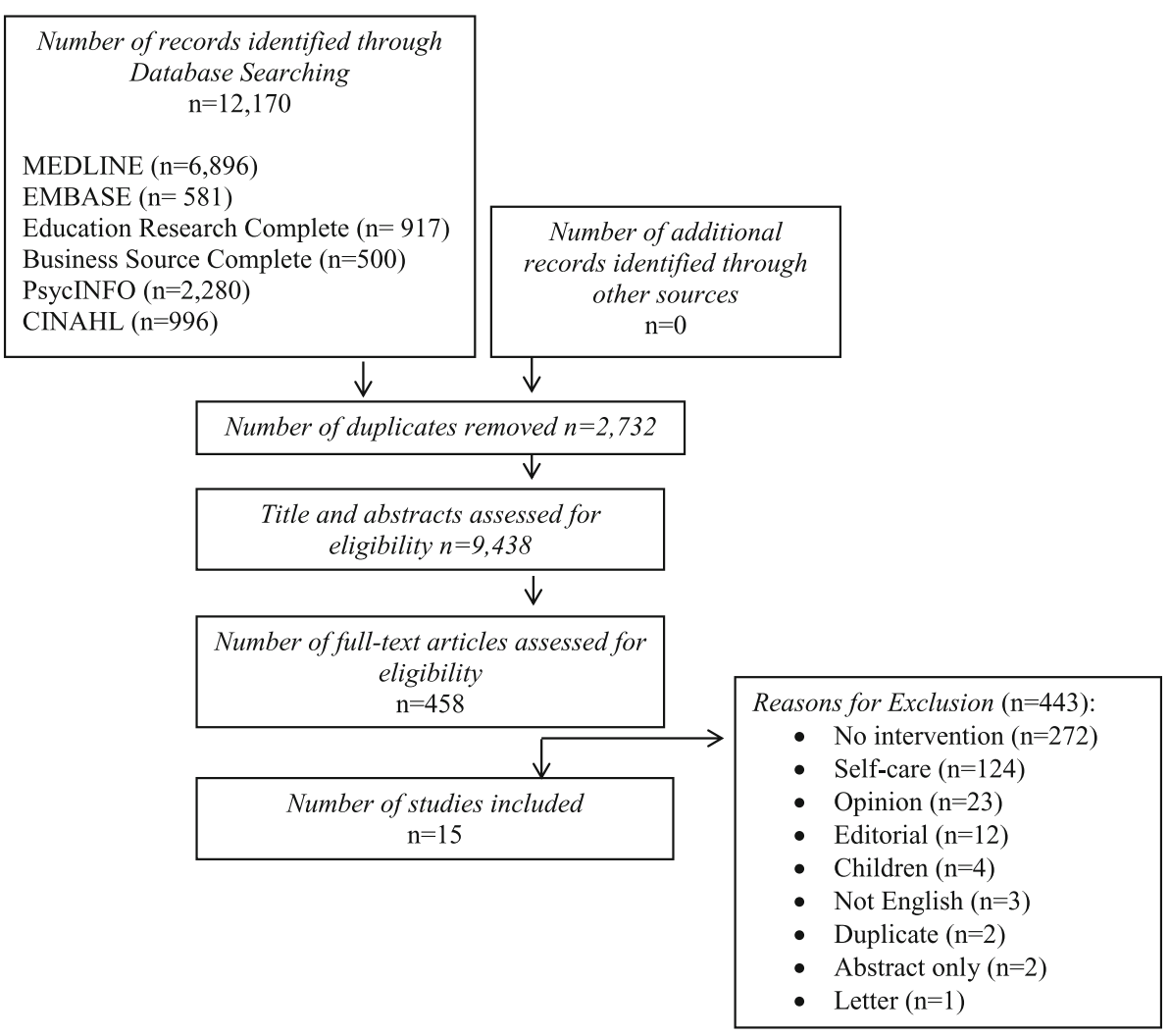

Fig. 1 Study flow

Table 1 Study characteristics

\begin{tabular}{ll}
\hline Study characteristics $(n=15)$ & Count (\%) \\
\hline Year of publication & $4(27)$ \\
$2001-2005$ & $3(20)$ \\
$2006-2011$ & $8(53)$ \\
$2012-2016$ & \\
Location of study & $7(7)$ \\
UK & $3(20)$ \\
US & $2(13)$ \\
Australia & $1(7)$ \\
Canada & $1(7)$ \\
New Zealand & $1(7)$ \\
Germany & \\
Study design & $9(60)$ \\
Qualitative & $3(20)$ \\
Mixed methods & $2(13)$ \\
Quantitative (non-randomized) & $1(7)$ \\
Randomized control trial &
\end{tabular}

published in Canada, New Zealand, and Germany $(n=1)$. Nine of the studies utilized qualitative research methodologies to address their research questions, followed by mixed methods $(n=3)$ and quantitative non-randomized designs $(n=2)$.

\section{Quality assessment}

Eight of the studies (7 qualitative and 1 quantitative) were deemed of high quality, and 3 studies (1 qualitative and 2 quantitative) were of medium quality. Factors that impacted the quality assessment were fairly consistent. The qualitative studies $(n=6)$ primarily did not discuss the role of the researcher (Q1.4), and two of the 2 quantitative research studies did not adequately report outcome data (Q2.3 and Q3.3). Four studies did not meet the initial screening questions and were not further assessed. Since we did not exclude based on quality studies not meeting the initial screening questions were not included in the presentation of outcomes (see Table 2).

\section{Study themes}

The included studies $(n=15)$ were themed into four categories by the research team including forums, patient instructors, workshops, and co-design. The first theme, forums, included five studies that highlight examples of 
Table 2 Mixed Methods Assessment Tool applied to included studies

Q1.1 Are the sources relevant? Q1.2 Is process for analyzing relevant? Q1.3 Is consideration given to how findings related to the context? Q1.4 Is consideration given to how findings relate to influence?

Q2.1 Is randomization clear? Q2.2 Is allocation concealment clear? Q2.3 Are there complete outcome data? Q2.4 Is there low dropout?

Q3.1 Is selection bias minimized? Q3.2 Are measurements appropriate for exposure \& outcomes? Q3.3 Are participants comparable? Q3.4 Are there complete outcome data \& response rate?

Q5.1 Is design relevant to address question? Q5.2* Is integration of data relevant? Q5.3*

Is appropriate consideration given to limitations?

\begin{tabular}{|c|c|c|c|c|c|c|c|c|c|}
\hline Theme & Author & Year & Design & Q1 & Q2 & Q1.1 & Q1.2 & Q1.3 & 1.4 \\
\hline $\begin{array}{l}\text { Patient } \\
\text { Instructor }\end{array}$ & Shah & 2005 & Qualitative & \begin{tabular}{|l|}
$\mathrm{Y}$ \\
\end{tabular} & \begin{tabular}{|l|} 
\\
$Y$
\end{tabular} & $\mathrm{Y}$ & $\mathrm{Y}$ & $\mathrm{Y}$ & - \\
\hline Forums & Marlett & 2015 & Qualitative & $\mathrm{Y}$ & $\mathrm{Y}$ & $\mathrm{Y}$ & $\mathrm{Y}$ & $\mathrm{Y}$ & - \\
\hline Forums & McElfish & 2016 & Qualitative & $\mathrm{Y}$ & $\mathrm{Y}$ & $\mathrm{Y}$ & $\mathrm{Y}$ & $\mathrm{Y}$ & $\mathrm{Y}$ \\
\hline Workshops & Hyde & 2016 & Qualitative & $\mathrm{Y}$ & $\mathrm{Y}$ & $\mathrm{Y}$ & $\mathrm{Y}$ & $\mathrm{Y}$ & $\mathrm{Y}$ \\
\hline Workshops & Boaz & 2016 & Qualitative & $\mathrm{Y}$ & $\mathrm{Y}$ & $\mathrm{Y}$ & $\mathrm{Y}$ & $\mathrm{Y}$ & - \\
\hline Workshops & Saunders & 2012 & Qualitative & $\mathrm{Y}$ & $\mathrm{Y}$ & $\mathrm{Y}$ & $\mathrm{Y}$ & $\mathrm{Y}$ & - \\
\hline Workshops & Boyd & 2012 & Qualitative & $\mathrm{Y}$ & $\mathrm{Y}$ & $\mathrm{Y}$ & $\mathrm{Y}$ & - & - \\
\hline $\begin{array}{c}\text { Patient } \\
\text { Instructor }\end{array}$ & Donaghy** & 2010 & Qualitative & - & - & - & - & - & - \\
\hline Forums & Oliver & 2001 & Qualitative & $\mathrm{Y}$ & $\mathrm{Y}$ & $\mathrm{Y}$ & $\mathrm{Y}$ & $\mathrm{Y}$ & - \\
\hline \multicolumn{2}{|r|}{ Article ID } & Year & Design & Q1 & Q2 & Q2.1 & Q2.2 & Q2.3 & Q2.4 \\
\hline $\begin{array}{c}\text { Patient } \\
\text { Instructor }\end{array}$ & Jha & 2015 & RCT & $\mathrm{Y}$ & $\mathrm{Y}$ & $\mathrm{Y}$ & $\mathrm{Y}$ & - & - \\
\hline \multicolumn{2}{|r|}{ Article ID } & Year & Design & Q1 & Q2 & Q3.1 & Q3.2 & Q3.3 & Q3.4 \\
\hline Workshops & Andejeski & 2002 & Quantitative (NR) & $\mathrm{Y}$ & $\mathrm{Y}$ & $\mathrm{Y}$ & $\mathrm{Y}$ & $\mathrm{Y}$ & $\mathrm{Y}$ \\
\hline Forums & Davis & 2001 & Quantitative (NR) & $\mathrm{Y}$ & $\mathrm{Y}$ & $\mathrm{Y}$ & - & - & $\mathrm{Y}$ \\
\hline \multicolumn{2}{|r|}{ Article ID } & Year & Design & Q1 & Q2 & Q5.1 & Q5.2 & Q5.3 & Q5.4 \\
\hline Forums & Shelton** & 2011 & Mixed Method & $\mathrm{Y}$ & - & - & - & - & - \\
\hline Workshops & Tischler** & 2010 & Mixed Method & - & $\mathrm{Y}$ & - & - & - & - \\
\hline Workshops & Langer** & 2016 & Mixed Method & - & - & - & - & - & - \\
\hline
\end{tabular}

*Both qualitative and quantitative components must be addressed

**Study not included in the presentation of outcomes

participants receiving some form of instruction. These studies included examples of patients participating in a 1-year training program to be able to conduct patient engagement research [32], a series of informal training opportunities injected throughout a health technology assessment project [33], informal training offered to patients during stakeholder meetings to assist in developing a shared understanding of patient-centered outcomes [34], a 3-day advocacy training course to build confidence among breast cancer patients [35], and a 19-h 3-month training course for lay trainers to become patient navigators [36]. The second theme, patient instructors, expanded our definition of investment and included three studies that provided patient and family members with an opportunity to give an investment of time, as in providing patients with the opportunity and space to participate in healthcare delivery/health system improvements through their participation in student training [37-39]. The third theme, workshops, included five studies that highlight how patients are learning skills to participate in certain tasks. These studies included examples of opportunities for patients to participate in workshops to understand the systematic review process [40], a general information workshop to understand the research needs of cancer patients [41], an orientation presentation to enable patients to participate in a scientific review process [42], a series of workshops to help patients develop a common definition of patient-centeredness [43], and a series of learning opportunities to evaluate a collaborative learning model [44]. Finally, the fourth theme, co-design, included two studies involving patients who were involved in co-designing service delivery improvements: the first study involved patients in implementing improvement projects within acute hospital settings [45] and the second study co-designed improvements within a breast cancer service project [46]. See Table 3 for a complete description of the included studies.

\section{Study outcomes}

Four of the 15 included studies explored the impact of the respective investments on increasing patient engagement in healthcare decision-making. Two studies discussed the impact of forums on patient involvement. The first study found that patients who had received this investment of training had significantly increased their involvement in advocacy activities, such as acting as a community board member [35]. The second study found that a 1-year training program increased the confidence 


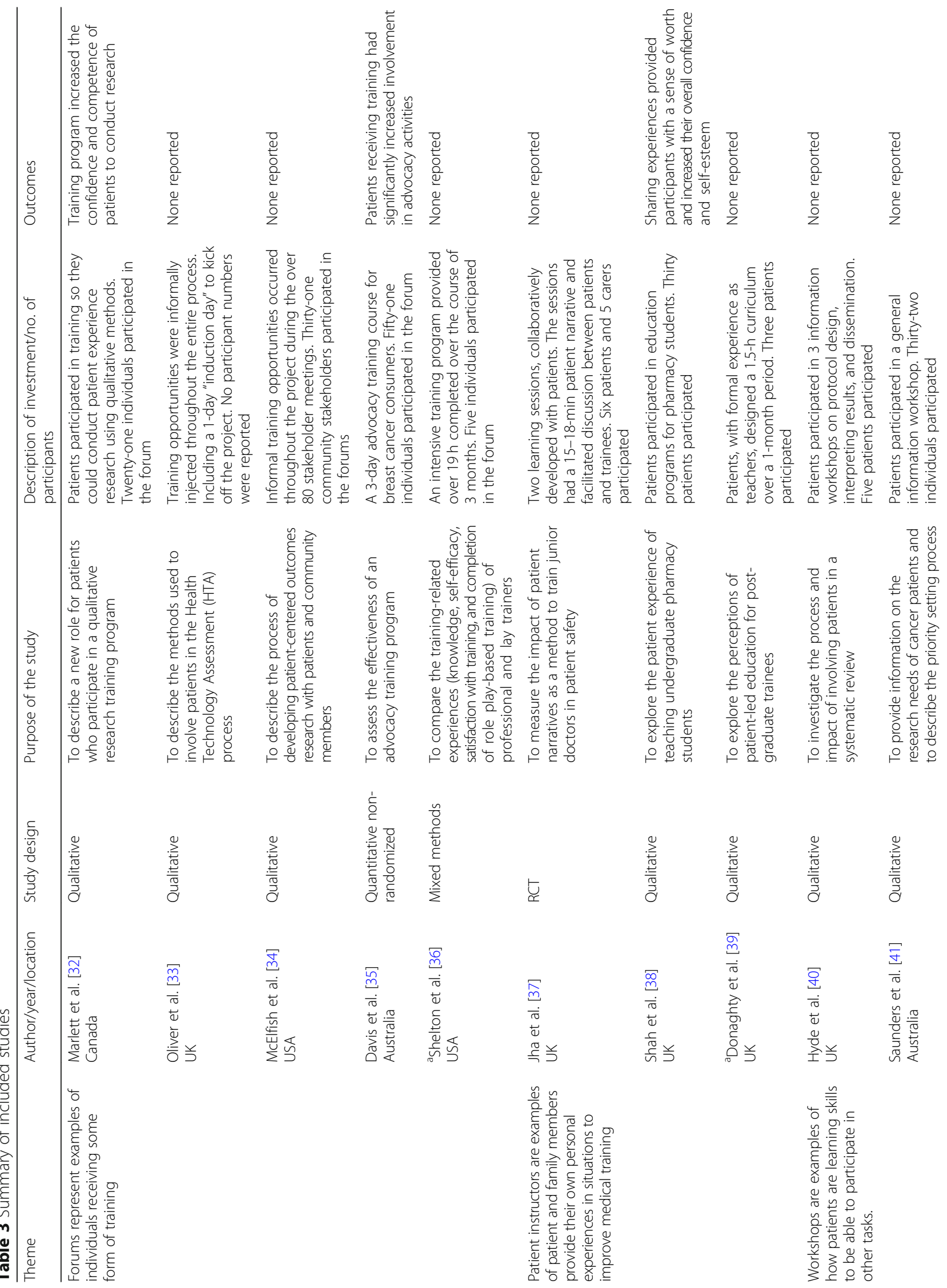




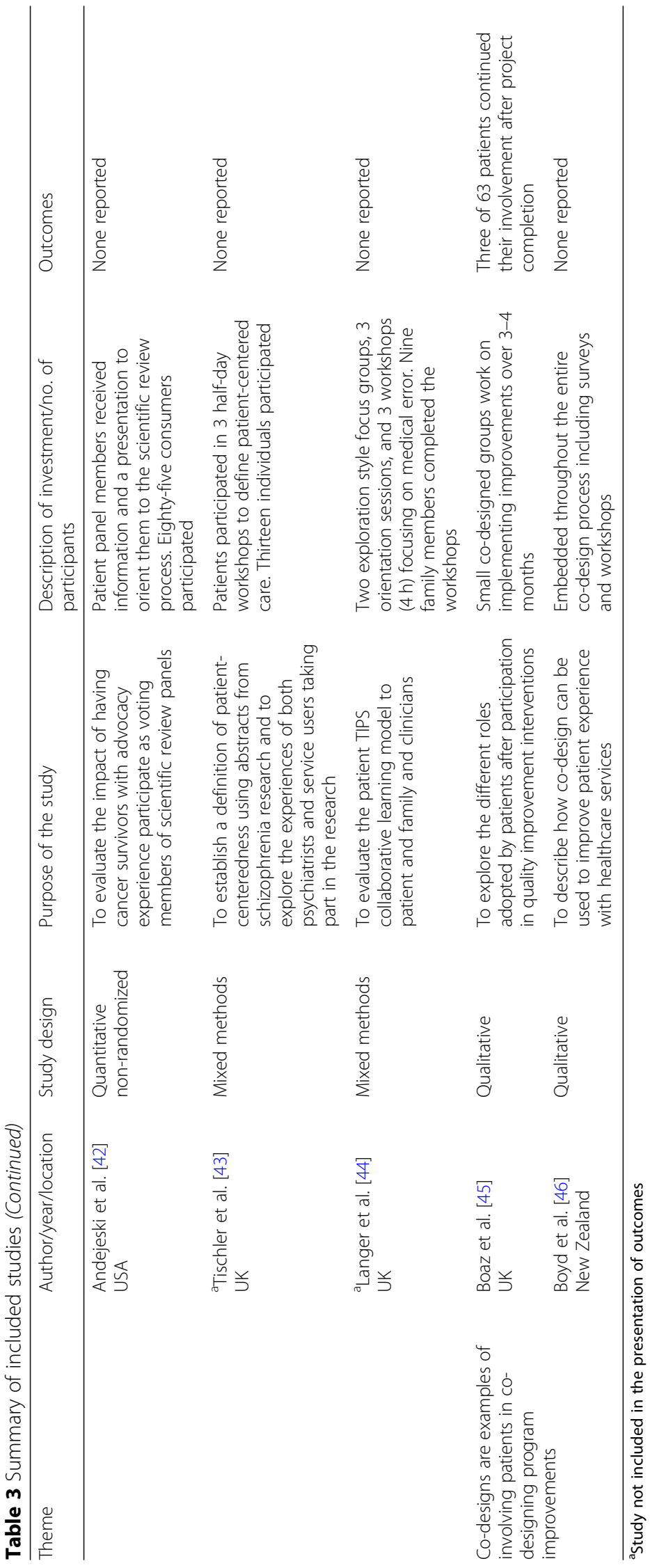


and competence of patients to conduct health research [32]. The third study discussed the impact of patient instructors and found that the opportunity to share the patient experience with pharmacy students provided participants with a sense of worth and increased their overall confidence and self-esteem [38]. Lastly, the fourth study discussed the impact of co-design on patient involvement finding three of 63 patients continued their involvement after the project was complete [45]. Overall, these studies found positive outcomes resulting from one of these four investments.

\section{Discussion}

Acknowledging the complexity of patient engagement, we undertook this scoping review to explore the nature and impact of investments implemented by health systems to build the capacity and ability of individuals to meaningfully participate in healthcare decision-making. We identified 15 diverse studies and four investment themes: (1) forums, (2) patient instructors, (3) workshops, and (4) co-design. Four of the 15 included studies evaluated the impact of programs designed to increase patient engagement in healthcare. The results of this scoping review indicate there is an opportunity for future research to further establish and evaluate programs that facilitate patient involvement. During this review, the authors noted that there were a number of gaps in the literature such as training to build additional competencies, such as governance experience; removing participation barriers for patients, such as providing financial assistance for expenses, including child care; and creating roles or opportunities for patients to develop new skills or further develop their existing skills.

This review is the first to comprehensively assess how health systems are investing in building the capacity and ability of patients. A number of benefits have been reported in previous studies that primarily focus on promoting self-efficacy and empowering and equipping patients with the skills and confidence to manage their own self-care [47-49]. Although enabling patient self-care is essential for the delivery of efficient and effective healthcare, opportunities exist to expand into other areas of patient engagement. The types of investments suggest there may be value in shifting the focus from patient engagement in self-care to an exploration of other ways in which health professionals and healthcare systems can benefit from engaging patients in healthcare governance and the establishment of system-level priorities.

While significant funding has been allocated to advance the inclusion of patients in healthcare decisionmaking, there is a lack of quality evidence to assess the transferability of various approaches to patient engagement in other settings. This lack of rigorous research may be contributing to the general absence of systemwide adoption of initiatives to encourage broader patient engagement in healthcare decision-making. There is a need to invest in interventions that evaluate the impact and effectiveness of these programs. Public-private partnerships such as the European Patients' Academy (EUPATI) provide training opportunities for patients to increase their capacity and capability to contribute to health research [50]. Although the mission of the EUPATI initiative is that these interventions will translate into a new paradigm of increased patient involvement across the entire health research spectrum, it is still early to tell [51]. The Health Technology Assessment International (HTAi) [52] and James Lind Alliance [53] have resources available for patients and the public but do not provide tailored training opportunities. Studies further exploring the impact of these efforts should be undertaken.

The manner in which we approached this review served a dual purpose of both assessing the extent of the literature on patient engagement and providing a real-world opportunity to develop the capacity of patients to participate in this type of research. The patient co-investigators experienced an increased level of confidence in their abilities to participate in a scoping review as a result of this experience. In addition, all three patient co-investigators have sought additional opportunities for their involvement.

\section{Strengths and limitations}

This study has strengths and limitations. We limited our search to English language peer-reviewed publications. As a result, it is possible that a search that sought to identify gray literature and research published in languages other than English may have yielded additional studies of relevance to this review. Due to the comprehensive search strategy, the volume of studies required us to limit publication dates of the included studies. While people have been thinking about engagement for over 50 years, it is important for programs that envision engagement beyond self-care be realized [2, 54]. This scoping review considered only investments from the patient perspective but it is also important to understand how health professionals are being supported to enable and support opportunities for patients. The inclusion of literature that focused on engagement strategies aimed at health professionals could have identified additional approaches to involving stakeholders in healthcare decision-making. Lastly, while the inclusion of patient co-investigators in the evidence synthesis could be perceived as design bias, we feel that the robust methodological processes we developed to conduct this review minimized any potential for bias, while supporting greater understanding and confidence among the patient co-investigators. 


\section{Conclusion}

While significant research exists that highlights how health systems are working with patients to better manage their own care, studies that explore other dimensions of patient engagement are largely absent. This study identified a few examples of how health systems are investing in building the capacity of patients. Creating opportunities for training and skill building, in all aspects of healthcare, enables patients to see first-hand the challenges faced by the healthcare system. This perspective helps to establish the role of the patient as a valued partner in healthcare decision-making. The results of this review suggest that achieving person-centered care may still be a long time away.

\section{Additional files}

Additional file 1: Final MEDLINE Search Strategy. (DOCX $15 \mathrm{~kb}$ )

Additional file 2: Data-charting (extraction) sheet. (XLSX 9 kb)

\section{Abbreviations}

PRISMA-P: Preferred Reporting Items for Systematic review and Meta-Analysis Protocols; PRISMA-SCR: PRISMA extension for scoping reviews; SPICE: Setting, population/perspective, intervention, comparison and evaluation; MMAT: Mixed Methods Appraisal Tool

\section{Acknowledgements}

The authors would like to thank Mark Hofmeister for his contribution to the Quality Assessment section of this paper.

\section{Funding}

Funding for this project was provided by Alberta Health Services and supported by a CIHR Barer-Flood Prize for Health Services and Policy Research (TN).

\section{Availability of data and materials}

All data generated or analysed during this study are included in this published article.

\section{Authors' contributions}

TLM had significant involvement in the design, acquisition, analysis, and interpretation of the data. DW, DH, DLL, TN, and NM provided guidance in the overall design and delivery of the research. KM, GW, SZ, and JMB were significantly involved in the acquisition and analysis. All the named authors were involved in revising the content, agree to take accountability for the integrity and accuracy of the work, and have read and approved the final manuscript.

\section{Ethics approval and consent to participate}

Not applicable

\section{Consent for publication}

Not applicable

\section{Competing interests}

The authors declare that they have no competing interests.

\section{Publisher's Note}

Springer Nature remains neutral with regard to jurisdictional claims in published maps and institutional affiliations.

\section{Author details}

${ }^{1}$ The Department Community Health Sciences, Teaching Research and Wellness Building, 3280 Hospital Drive NW, Calgary, Alberta T2N 4N1, Canada. ${ }^{2} \mathrm{O}$ 'Brien Institute for Public Health, Teaching Research and Wellness Building,
3280 Hospital Drive NW, Calgary, Alberta T2N 4N1, Canada. ${ }^{3}$ Patient Co-investigators, Community Health Sciences, University of Calgary, Alberta, Canada. ${ }^{4}$ Faculty of Nursing, University of Calgary in Qatar, PO Box 23133, Doha, Al Rayayan Al Forousiya, Qatar. ${ }^{5}$ Haskayne School of Business, 2500 University Dr. NW, Calgary, Alberta T2N 1N4, Canada. ${ }^{6}$ Health Sciences Library, University of Calgary, 3330 Hospital Drive NW, Calgary, Alberta T2N $4 \mathrm{~N}$, Canada.

Received: 2 October 2018 Accepted: 22 March 2019

Published online: 18 April 2019

\section{References}

1. Balint E. The possibilities of patient-centered medicine. J R Coll Gen Pract. 1969;17(82):269-76.

2. Carman $\mathrm{KL}$, et al. Patient and family engagement: a framework for understanding the elements and developing interventions and policies. Health Aff. 2013;32(2):223-31.

3. Gallivan J, et al. The many faces of patient engagement. J Participatory Med. 2012;4:e32. Retrieved from: https://participatorymedicine.org/journal/ evidence/research/2012/12/26/the-many-faces-of-patientengagement/4/.

4. Garces JP, et al. In: M. Clinic, editor. Eliciting patient perspective in patientcentered outcomes research: a meta narrative systematic review. Rochester: Patient-Centered Outcomes Research Institute; 2012.

5. Sherman R. O. \& Hilton N. The patient engagement imperative. American Nurse Today. 2014;9(2). Retrieved from https://www.americannursetoday. $\mathrm{com} /$ ?s=the+patient+engagement+imperative.

6. Taylor D, et al. Effectiveness of tai chi as a community-based falls prevention intervention: a randomized controlled trial. J Am Geriatr Soc. 2012;60(5):841-8.

7. Baker GR. In: C.F.o.H. Improvement, editor. Evidence boost: a review of research highlighting how patient engagement contributes to improved care; 2014.

8. Sarrami $P$, et al. Consumer and community engagement: a review of the literature. Australia: Australian Institute of Health Innovation; 2012.

9. Buck D, et al. From plans to actions in patient and public involvement: qualitative study of documented plans and the accounts of researchers and patients sampled from a cohort of clinical trials. BMJ Open. 2014;4(12):1-19. doi: https://doi.org/10.1136/bmjopen-2014-006400.

10. Charmel PA, Frampton SB. Building the business case for patient-centered care. Healthcare Financ Manage. 2008;62(3):80-5.

11. Abelson J, et al. In: C.H.S.R. Foundation, editor. Effective strategies for interactive public engagement in the development of healthcare policies and programs. New Brunswick: Publisher is Canadian Health Services Research Foundation; 2010. Online access: https://www.cfhi-fcass.ca/SearchResultsNews/ 11-03-09/3b6b5648-d03f-4d7d-aed6-8df557e600ca.aspx.

12. Coulter A. Engaging patient in healthcare. United Kingdom: Ashford Colout Press; 2011.

13. Brett J, et al. The PIRICOM study: a systematic review of the conceptualisation, measurement, impact and outcomes of patients and public involvement in health and social care research. Warwick: University of Warwick; 2010.

14. Sustania. Person-centered care: co-creating a healthcare sector for the future. United Kingdom: Sustania; 2015. Online access: https://issuu.com/sustainia/ docs/personcentredcare.

15. Hibbard HJ. Engaging health care consumers to improve the quality of care Med Care. 2003:41(1 Suppl):I-61--1-70.

16. Barello S, Graffigna G, Vegni E. Patient engagement as an emerging challenge for healthcare services: mapping the literature. Nurs Res Pract. 2012;2012:7

17. Cottam H, Leadbeater C. In: D. Council, editor. Red paper 01 health: co-creating services. United Kingdom: Design Council; 2004. Accessed online: https://www.designcouncil.org.uk/sites/default/files/asset/ document/red-paper-health.pdf.

18. Wood SB, Henderson S. Realising the value: what the system can do. United Kingdom: Nesta; 2016. Online access: https://www.nesta.org.uk/report/whatthe-system-can-do-the-role-of-national-bodies-in-realising-the-valueofpeople-and-communities-in-health-and-care/.

19. Lancaster $\mathrm{K}$, et al. The use and effects of electronic health tools for patient self-monitoring and reporting of outcomes following medication use: systematic review. J Med Internet Res. 2018;20(12):e294. doi: https://doi. org/10.2196/jmir.9284

20. Cassidy BP, et al. Barriers to education and shared decision making in the chronic kidney disease population: a narrative review. Can J Kidney Health Dis. 2018;5:1-11. doi: https://doi.org/10.1177/2054358118803322. 
21. Arksey H, O'Malley L. Scoping studies: towards a methodological framework. Int J Soc Res Methodol. 2005;8(1):19-32.

22. Levac D, Colquhoun H, O'Brien K. Scoping studies: advancing the methodology. Implement Sci. 2010:5(69):1-9. doi: https://doi.org/10.1186/1748-5908-5-69.

23. Pham MT, et al. A scoping review of scoping reviews: advancing the approach and enhancing the consistency. Res Synth Methods. 2014;5(4):371-85.

24. Daudt HML, van Mossel C, Scott SJ. Enhancing the scoping study methodology: a large, inter-professional team's experience with Arksey and O'Malley's framework. BMC Med Res Methodol. 2013;13:48.

25. Booth A. Clear and present questions: formulating questions for evidence based practice. Library Hi Tech. 2006;24(3):355-68.

26. Tricco A, et al. PRISMA extension for scoping reviews (PRISMA-SCR): checklist and explanation. Ann Intern Med. 2018;169(7):467.

27. Bate $P$, Robert G. Bringing user experience to healthcare improvement: the concepts. Methods and practices of experience-based design. Oxford; New York: Radcliff Publishers; 2007.

28. Sanders EBN, Stappers PJ. Co-creation and the new landscapes of design. CoDesign. 2008;4(1):5-18.

29. Pluye, P., et al. Mixed Methods Appraisal Tool. 2011 [cited 2017 September 17]; Available from: http://mixedmethodsappraisaltoolpublic.pbworks.com/w/file/ fetch/84371689/MMAT\%202011\%20criteria\%20and\%20tutorial\%202011-0629updated2014.08.21.pdf.

30. Pace $\mathrm{R}$, et al. Testing the reliability and efficiency of the pilot Mixed Methods Appraisal Tool (MMAT) for systematic mixed studies review. Int J Nurs Stud. 2012;49(1):47-53. doi: https://doi.org/10.1016/j.jjnurstu.2011.07.002.

31. Glaser BG. The constant comparative method of qualitative analysis. Soc Probl. 1965:12(4):436-45.

32. Marlett $\mathrm{N}$, et al. Building new roles and relationships in research: a model of patient engagement research. Qual Life Res. 2015;24(5):1057-67.

33. Oliver $\mathrm{S}$, et al. Involving consumers in a needs-led research programme: a pilot project. Health Expect. 2001;4(1):18-28.

34. McElfish PA, et al. Engagement practices that join scientific methods with community wisdom: designing a patient-centered, randomized control trial with a Pacific Islander community. Nurs Inq. 2016:20.

35. Davis C, Salo L, Redman S. Evaluating the effectiveness of advocacy training for breast cancer advocates in Australia. Eur J Cancer Care. 2001;10(2):82-6.

36. Shelton $\mathrm{RC}$, et al. Training experiences of lay and professional patient navigators for colorectal cancer screening. J Cancer Educ. 2011;26(2): 277-84.

37. Jha $V$, et al. Patients as teachers: a randomised controlled trial on the use of personal stories of harm to raise awareness of patient safety for doctors in training. BMJ Qual Saf. 2015;24(1):21-30 10p.

38. Shah R, Savage I, Kapadia S. Patients' experience of educating pharmacy undergraduate students. Pharm Educ. 2005;5(1):61-7.

39. Donaghy F, Boylan $\mathrm{O}$, Loughrey C. Using expert patients to deliver teaching in general practice. Br J Gen Pract. 2010;60(571):136-9.

40. Hyde $C$, et al. Process and impact of patient involvement in a systematic review of shared decision making in primary care consultations. Health Expect. 2017;20(2):298-308 DOl: https://doi.org/10.1111/hex.12458.

41. Saunders C, Crossing S. Towards meeting the research needs of Australian cancer consumers. BMC Res Notes. 2012;5:667.

42. Andejeski $Y$, et al. Quantitative impact of including consumers in the scientific review of breast cancer research proposals. J Womens Health Gender Based Med. 2002;11(4):379-88 10p.

43. Tischler $V$, et al. Involving patients in research: the challenge of patientcentredness. Int J Soc Psychiatry. 2010;56(6):623-33.

44. Langer $T$, et al. Patients and families as teachers: a mixed methods assessment of a collaborative learning model for medical error disclosure and prevention. BMJ Qual Saf. 2016;25(8):615 DOl: https://doi.org/10.1136/bmjqs-2015-004292.

45. Boaz A, et al. What patients do and their impact on implementation. J Health Organ Manage. 2016;30(2):258-78.

46. Boyd H, et al. Improving healthcare through the use of co-design. N Z Med J. 2012;125(1357):76-87.

47. Wakefield D, et al. Patient empowerment, what does it mean for adults in the advanced stages of a life-limiting illness: a systematic review using critical interpretive synthesis. Palliative Medicine. 2018;32(8):1288-304 DOl: https://doi.org/10.1177/0269216318783919.

48. Hood KK, et al. Preventing diabetes distress in adolescents with type 1 diabetes: results one year after participation in the STePS program. Diabetes Care. 2018;41(8):1623-30 DOl: https://doi.org/10.2337/dc17-2556.
49. Riese $C$, et al. Effectiveness of a standardized patient education program on therapy-related side effects and unplanned therapy interruptions in oral cancer therapy: a cluster-randomized controlled trial. Support Care Cancer. 2017;25(11):3475-83.

50. European Patients' Academy. What is EUPATI? 2019 [cited 2019 January 23]; Available from: https://www.eupati.eu/what-is-eupati/.

51. Pushparajah D, Geissler J, Westergaard N. EUPATI: collaboration between patients, academia and industry to champion the informed patient in the research and development of medicines. J Med Dev Sci. 2015;1(1):74-80.

52. HTAi. For patient groups and individual patients. 2019 [cited 2019 January 23]; Available from: https:/htai.org/interest-groups/pcig/resources/for-patients-andpatient-groups/.

53. James Lind Alliance. The James Lind Alliance. 2019 [cited 2019 January 23]; Available from: http://www.jla.nihr.ac.uk.

54. Carman K. Workman K. Engaging patients and consumers in research evidence: applying the conceptual model of patient and family engagement. Patient Educ Couns. 2016;100(2017):25-39.
Ready to submit your research? Choose BMC and benefit from:

- fast, convenient online submission

- thorough peer review by experienced researchers in your field

- rapid publication on acceptance

- support for research data, including large and complex data types

- gold Open Access which fosters wider collaboration and increased citations

- maximum visibility for your research: over $100 \mathrm{M}$ website views per year

At $\mathrm{BMC}$, research is always in progress.

Learn more biomedcentral.com/submissions 